\title{
Cell-mediated and humoral immune profile to hydatidosis among naturally infected farm animals
}

\author{
Faten A. M. Abo-Aziza ${ }^{1}$, Seham H. M. Hendawy ${ }^{1}$, Samah S. Oda ${ }^{2}$, Dina Aboelsoued ${ }^{1}$ and Eman E. El Shanawany ${ }^{1}$
}

1. Department of Parasitology and Animal Diseases, Veterinary Research Division, National Research Centre, Cairo, Egypt; 2. Department of Pathology, Faculty of Veterinary Medicine, Alexandria University, Edfina, Egypt.

Corresponding author: Faten A. M. Abo-Aziza, e-mail: faten.aboaziza@gmail.com

Co-authors: SHMH: shendawy2006@yahoo.com, SSO: samahoda@gmail.com, DA: dr.dina.aboelsoued@gmail.com, EEE: ee.elshanawany@hotmail.com

Received: 25-09-2019, Accepted: 23-12-2019, Published online: 31-01-2020

doi: www.doi.org/10.14202/vetworld.2020.214-221 How to cite this article: Abo-Aziza FAM, Hendawy SHM, Oda SS, Aboelsoued D, El Shanawany EE (2020) Cell-mediated and humoral immune profile to hydatidosis among naturally infected farm animals, Veterinary World, 13(1): 214-221.

\begin{abstract}
Background and Aim: Cystic echinococcosis (CE) is a widespread parasitic disease caused by Echinococcus granulosus tapeworm infect different intermediate hosts including sheep, cattle, and camels. The intermediate host's immune response to the hydatid cyst is still conflict and complex. The current study was designed to evaluate the immune response in sera of hydatid naturally infected sheep, cattle, and camels in the form of features of inflammatory cell infiltrations, levels of Th1 and Th2 cytokines, besides the humoral specific immunoglobulin $\mathrm{G}(\mathrm{IgG})$ responses.
\end{abstract}

Materials and Methods: Thirty-nine sheep, 74 cattle, and 79 camels' sera were collected and considered as CE naturally infected and ten samples from each species were graded as non-infected. Lung specimens were collected for histopathological examination. The quantitative concentrations of tumor necrosis factor- $\alpha$, interleukin (IL)- 6 , IL-4, and IL-10 were determined. Different antigens were prepared from hydatid cyst; hydatid cyst fluid of lung origin hydatid cyst fluid of liver origin, hydatid cyst protoscoleces of lung origin (HCP-g), hydatid cyst protoscoleces of liver origin, hydatid cyst germinal layer of lung origin, and hydatid cyst germinal layer of liver origin; and characterized by gel electrophoresis and Western blotting analysis. The total specific IgG level against E. granulosus infection was measured using an indirect enzyme-linked immunosorbent assay.

Results: The results indicated that the cellular immune response in the infected tissues was characterized by inflammatory cell penetration. The pro-inflammatory Th1 cytokine profile was predominant in infected animals in comparison with non-infected ones. However, the humoral immune response was seen as a high level of IgG in infected animals. The presented data approved that the HCP-g antigen could be considered as a delegate antigen for all other prepared antigens with an immunoreactive band at molecular weights $32 \mathrm{kDa}$.

Conclusion: This study provides a fundamental insight into the events that manipulate cellular and humoral immune profiles in an intermediate host; sheep, cattle, and camel that naturally infected with CE. Hence, it was concluded that CE is a constant disease and confirm the reactivity Th1 in combating hydatid cyst. Besides, it could lead to the activation of the humoral immune response in the form of a high level of $\mathrm{IgG}$.

Keywords: cell-mediated immune response, humoral immune response, hydatidosis.

\section{Introduction}

Cystic echinococcosis (CE) or hydatidosis is a worldwide parasitic disease caused by the cestode Echinococcus granulosus. Hydatidosis is a silent cyclozoonotic disease of humans and herbivore animals causing high morbidity and mortality rate [1]. The larval stage of E. granulosus has a complex life cycle which alternates between definitive carnivore hosts such as dogs and other canids [2] and intermediate hosts including herbivore animals such as cattle, pigs, buffaloes, camels, sheep, and goats [3].

Copyright: Abo-Aziza, et al. Open Access. This article is distributed under the terms of the Creative Commons Attribution 4.0 International License (http://creativecommons.org/licenses/ by/4.0/), which permits unrestricted use, distribution, and reproduction in any medium, provided you give appropriate credit to the original author(s) and the source, provide a link to the Creative Commons license, and indicate if changes were made. The Creative Commons Public Domain Dedication waiver (http:// creativecommons.org/publicdomain/zero/1.0/) applies to the data made available in this article, unless otherwise stated.
Furthermore, human may be infected as an intermediate host accidentally by contaminated food or water or by direct contact with infected dog feces [4]. Activated oncosphere larvae are released from hatched eggs at the gastrointestinal tract of the intermediate hosts. They penetrate the intestinal wall to reach the bloodstream and eventually, reside in the internal organs where they mature to form hydatid cysts [5]. The hydatid cysts develop in different viscera, especially in the liver and lungs, which are common places for cyst formation [6] and gradually grow from $1 \mathrm{~cm}$ to $5 \mathrm{~cm}$ a year [7]. The hydatid cysts are usually unilocular, fluid-filled bladder structures which consist of two layers; outer laminated layer surrounded by fibrous host tissue (pericyst) and innermost germinal layer where brood capsules and protoscoleces may bud from germinal membrane [8]. The host pericyst layers that surround the tissue of the parasite are considered as part of the cyst structures. These layers 
are of host origin and have an important role in the immunological response against the parasite [9]. The definitive hosts are infected after ingesting offal containing fertile hydatid cysts with viable protoscoleces which are released and reach the small intestine where they develop to adult worm after 4-5 weeks [9]. Recent clinical cyst classifications have underlined that hydatid cysts are morphologically different [8].

The establishment of hydatid cysts within the intermediate host occurs in long-term growth, so different immune mechanisms are induced during host-parasite interplay [10]. The early immune response toward CE was found to be not successful in preventing the infection and this implies the existence of elaborated by E. granulosus [11]. E. granulosus displays different immunological relationships with its hosts, therefore, great efforts have been invested to understand the immunobiology of the parasite in the intermediate host [7]. T-helper is the main immunocompetent cells by secretion of immune mediators Th1/Th2 [12]. The early stage of CE is characterized by immunoglobulin $\mathrm{G}(\mathrm{IgG})$ response that plays a crucial role in the killing of larval metacestode [10]. In addition, the host immune responses usually depend on infiltrated cells and low-level of polarized Th1 responses with low production of pro-inflammatory cytokines. However, Th2 polarized immune response produces anti-inflammatory cytokine with the progression of the cyst [13]. Moreover, interleukin (IL)-10 orchestrates the chronic stage of CE which is modulated by protoscoleces developmental stages [8]. Furthermore, the cellular inflammatory infiltrations including neutrophils, lymphocytes, and macrophages are considered as a characteristic feature of echinococcal infection [10]. Moreover, CE infection is remarkable with mixed Th1/Th2 polarized cytokines [8]. Rostami-Rad et al. [14] found that the Th1-type cytokine profile was predominant at the early post-infection phase (3-4 weeks), however, the shift to Th2-type cytokine appeared in the $4^{\text {th }}$ week and this suggests that the shift in Th1-type cytokine profile toward Th2-type explains that $\mathrm{CE}$ is a constant disease and confirm the reactivity Th1 in combating E. granulosus infection.

In Egypt, CE exists in various intermediate hosts, including cattle, buffaloes, sheep, and camels [15]. Unfortunately, there are limited reports about the host-parasite interplay and immunopathogenesis for the course of the infection in naturally infected animals. Understanding the immune response against this disease is urgently needed to find new biomarkers for diagnostic and prognostic purposes that will be a benefit in the treatment and control of such E. granulosus infections in farm animals.

The current study was designed to evaluate the immune response in sera of hydatid naturally infected sheep, cattle, and camels in the form of features of inflammatory cell infiltrations, evaluate levels of Th1 and Th2 cytokines, besides the humoral specific
$\mathrm{IgG}$ responses. Furthermore, the characterization of hydatid cyst different prepared antigens; protoscoleces, hydatid cyst fluid, and germinal layer of camel origin was done as an example.

\section{Materials and Methods}

\section{Ethical approval}

This study was approved by the Medical Research Ethics Committee of National Research Centre, Egypt (Number: 16230). The experiments were conducted according to the guidelines established by the International Animal Ethics Committee and according to the local laws and regulations.

\section{Animals and samples collection}

Tissue samples with their corresponding blood were collected from 397 sheep, 401 cattle, and 341 camels presented for slaughter at El-Warak, El-Basatin, and El-Moneibs laughterhouses from April 2017 to June 2018. All samples of each species were classified into two groups infected and non-infected according to postmortem examination. Thirty-nine sheep, 74 cattle, and 79 camels were considered as naturally infected with $\mathrm{CE}$ and ten animals from each species were graded as non-infected based on the absence of visible CE infection and clinical signs. Sera were aliquoted and stored at $-20^{\circ} \mathrm{C}$ until use. Liver and lung specimens were collected and rapidly fixed for histopathological examination. Hydatid cysts of camel origin were collected in phosphate-buffered saline (PBS) $\mathrm{PH}, 7.4$ for the preparation of antigens.

\section{Histopathological examination}

Lung tissue specimens from infected animals were fixed rapidly in $10 \%$ neutral buffered formalin for about $24 \mathrm{~h}$. Fixed specimens were trimmed, washed, dehydrated in ascending grades of ethyl alcohol, cleared in xylene, and embedded in paraffin wax. Then, thin sections of 4-5 $\mu \mathrm{m}$ thickness were performed and stained with hematoxylin and eosin for general microscopic examination [16].

Evaluation of Th1-polarized cytokine (tumor necrosis factor [TNF]- $\alpha$, IL-6), and Th2-polarized cytokine (IL-4, and IL-10)

Sandwich enzyme-linked immunosorbent assay (ELISA) was utilized to measure serum cytokine levels. TNF- $\alpha$, IL-6, IL-4, and IL-10 concentrations were determined with commercially available kits (Sigma-Aldrich, USA) following the manufacturer's instructions. The color change was measured by a spectrophotometer at a wavelength of $450 \mathrm{~nm}$.

\section{Preparation of hydatid cyst antigens}

Collected hydatid cysts from infected lungs and livers of the slaughtered camels were used for preparing different hydatid cyst antigens; hydatid cyst fluid of lung origin (HCF-g), hydatid cyst fluid of liver origin (HCF-v), hydatid cyst protoscoleces of lung origin (HCP-g), hydatid cyst protoscoleces of liver origin (HCP-v), hydatid cyst germinal layer of lung origin (HCG-g), and hydatid cyst germinal layer 
of liver origin (HCG-v) [17]. Hydatid cyst fluid was aseptically aspirated and centrifuged at $5000 \times g$ for $20 \mathrm{~min}$ in a cooling centrifuge. The supernatants were collected, dialyzed, and stored as HCF-g and HCF-v antigens at $-20^{\circ} \mathrm{C}$. Protoscoleces were obtained from the sediment of collected cyst fluid. They were washed 3 times with PBS, pH 7.2, and resuspended in PBS. The protoscoleces were exposed to three cycles of freezing, thawing, and sonication ( $1 \mathrm{~min}$ and 0.5 amplitude), then they were microscopically checked until no intact protoscoleces were detected. Then, the antigens were centrifuged at $10,000 \times g$ for $30 \mathrm{~min}$ and supernatants were collected and stored as HCP-g and HCP-v antigens at $-20^{\circ} \mathrm{C}$. Germinal layers of hydatid cysts were separated, washed several times and homogenized in PBS, pH 7.2. The homogenate was sonicated for three cycles ( $1 \mathrm{~min}$ and 0.5 amplitude) and centrifuged at $10,000 \times \mathrm{g}$ for $30 \mathrm{~min}$ in a cooling centrifuge. The supernatants were aspirated and stored as HCG-g and HCG-v antigens at $-20^{\circ} \mathrm{C}$. The total protein content of different hydatid cyst antigens was measured according to Lowry et al. [18].

\section{Gel electrophoresis and Western blotting analysis}

All the hydatid cyst prepared antigens were individually fractionated in four $12 \%$ sodium dodecyl sulfate-polyacrylamide gel electrophoresis (SDS-PAGE) using pre-stained molecular weights (MW) protein marker 16-270 kDa (GeneDirex, USA). After SDSPAGE, one gel was stained with Coomassie Brilliant Blue R-250 dye and analyzed using a gel documentation system [19]. The others were blotted on 0.45 nitrocellulose membranes [20]. Briefly, the blocked membranes with $1 \%$ dry skimmed milk/Tris-buffered saline (TBS) were incubated overnight with positive naturally infected sera from camel, cattle, and sheep origins at dilution $1: 50$ in $\mathrm{TBS} / 0.5 \%$ bovine serum albumin (BSA) against all prepared antigens. The nitrocellulose sheets were probed for $1 \mathrm{~h}$ with protein-A peroxidase conjugate at 1:2500 diluted with $0.5 \%$ BSA/TBS buffer. The substrate solution (1-chloronaphthol, Sigma-Aldrich, USA) was used for 20 min to develop the immunogenic bands. Then, the nitrocellulose membranes were photographed and analyzed using Molecular Imager Gel Doc ${ }^{\mathrm{TM}} \mathrm{XR}+$ with Image Lab Software (Bio-Rad, California, USA).

\section{Diagnostic accuracy of the selected antigen}

The prevalence of hydatid cyst infection, sensitivity, and specificity of HCP-g antigen was calculated in contrast to the parasitological investigation of the examined camel sera [12].

\section{E. granulosus specific IgG assay}

Evaluation of $\operatorname{IgG}$ antibody levels against E. granulosus infection was performed using indirect ELISA (I-ELISA). Checkerboard titrations were used for the determination of the optimal concentration of antigens, tested sera dilutions, and conjugate dilution. The assay was performed according to the method previously described [21]. Briefly, the plates were coated with diluted antigen at the concentration of $4 \mu \mathrm{g} / \mathrm{ml}$ in coating buffer; carbonate-bicarbonate buffer, $\mathrm{pH}$ 9.6; $(100 \mu 1 /$ well $)$, and incubated at $4{ }^{\circ} \mathrm{C}$ overnight. The unbinding sites were blocked using 1\% BSA in coating buffer then incubated for $1 \mathrm{~h}$ at room temperature. After washing, $100 \mu \mathrm{l}$ diluted sera (1:50) of samples in $0.05 \%$ PBS-T-20 were added and incubated for $2 \mathrm{~h}$ at $37^{\circ} \mathrm{C}$. The plates were washed and protein-A horseradish peroxidase conjugate was added $(100 \mu 1 /$ well $)$ at dilution $1: 1000$ and incubated for $1 \mathrm{~h}$ at $37^{\circ} \mathrm{C}$. Then, the substrate orthophenylenediamine was added and the optical densities (OD) were read at a wavelength of $450 \mathrm{~nm}$ with an ELISA reader (BIO-TEK, INC, ELx, 800UV, USA). The cutoff point of OD values was determined as described by Almazán et al. [22]. Sera obtained from healthy; non-infected camels were used in the assay as a negative control.

\section{Statistical analysis}

The data were analyzed using a one-way analysis of variance. Results were expressed as mean \pm standard deviation and values of $\mathrm{p}<0.05$ and $\mathrm{p}<0.01$ were considered statistically significant and highly significant respectively. The curves were represented by MEDCALC easy-to-use statistical software.

\section{Results}

As shown in Figure-1, hydatid cyst of camel showing aspiration of the fluid from the cyst (Figure-1a), opened evacuated hydatid cyst germinal layer of cyst wall. The cyst wall consisted of an outer thick fibrous layer and an inner thin germinal layer (Figure-1b) and opened hydatid cyst with a semi-solid matrix (Figure-1c). The hydatid cyst was full of scolices and membranes which replace the hydatid liquid. Germinal layer (Figure-1d and e) and tissue from hydatid infected lung of cattle (Figure-1f).

\section{Histopathological findings}

Lungs of camels had marked hydatid cysts that were consisted of the outer laminated layer and fibrous layer penetrated with inflammatory cells. The surrounding pulmonary alveoli were collapsed and some were emphysematous. Scoleces were found in alveoli (Figure-2a) with mononuclear inflammatory cells and few multinucleated giant cells aggregation. Furthermore, there were severe congestion, hemorrhage, and edema (Figure-2b). One cyst was suppurated (Figure-2c) and others were caseated (Figure-2c).

\section{Th1/Th2-type cytokine profile in hydatid naturally infected-animals}

The serum level of Th-1 pro-inflammatory cytokines, TNF- $\alpha$ and IL- 6 and Th- 2 anti-inflammatory cytokines, IL-4 and IL-10, in all the animals, was determined by sandwich ELISA, and the results obtained are presented in Table- 1 . The data revealed that infected sheep showed significantly a higher serum level of TNF- $\alpha$ and IL- 6 in comparison with the corresponding non-infected ones. However, IL-4 


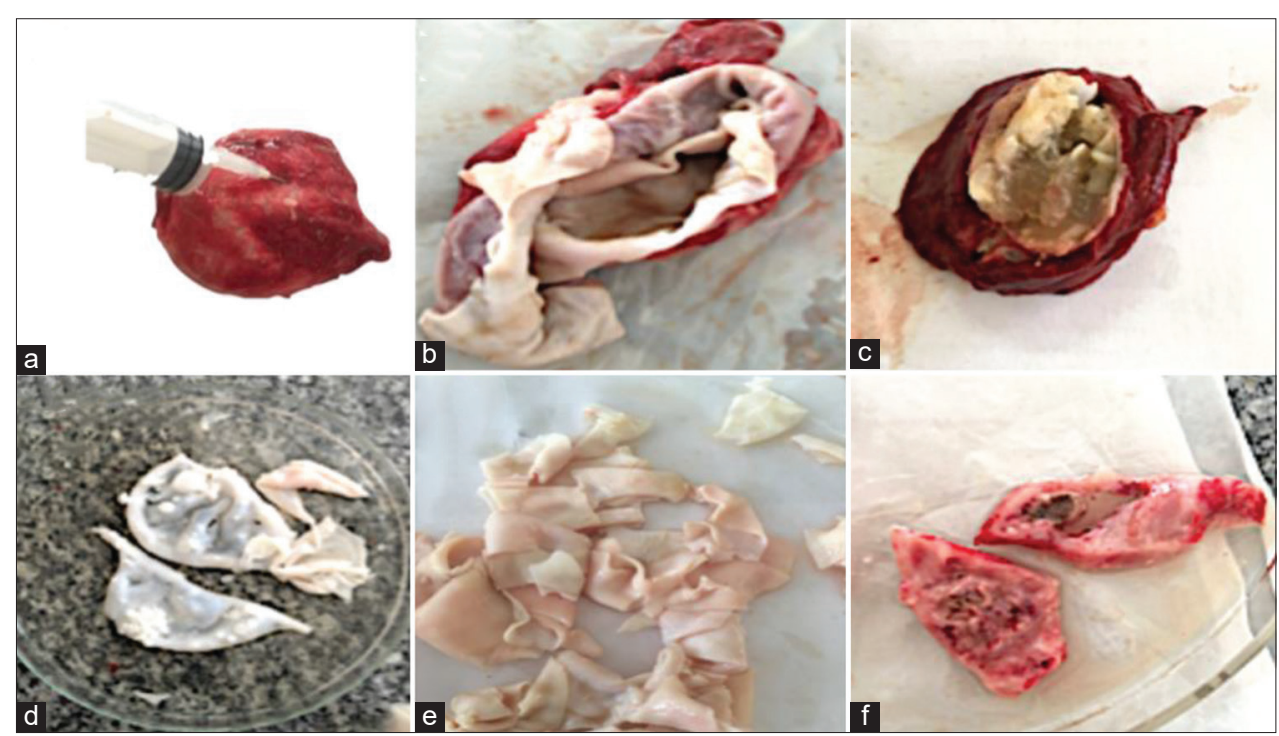

Figure-1: Hydatid cyst of camel showing aspiration of the fluid from the cyst (a), opened evacuated hydatid cyst germinal layer of cyst wall that consisted of an outer thick fibrous layer and an inner thin germinal layer (b), opened hydatid cyst of cattle with semi-solid matrix (c), a germinal layer of hydatid cyst of camel liver ( $d$ and e), tissue from hydatid infected lung of sheep $(f)$.

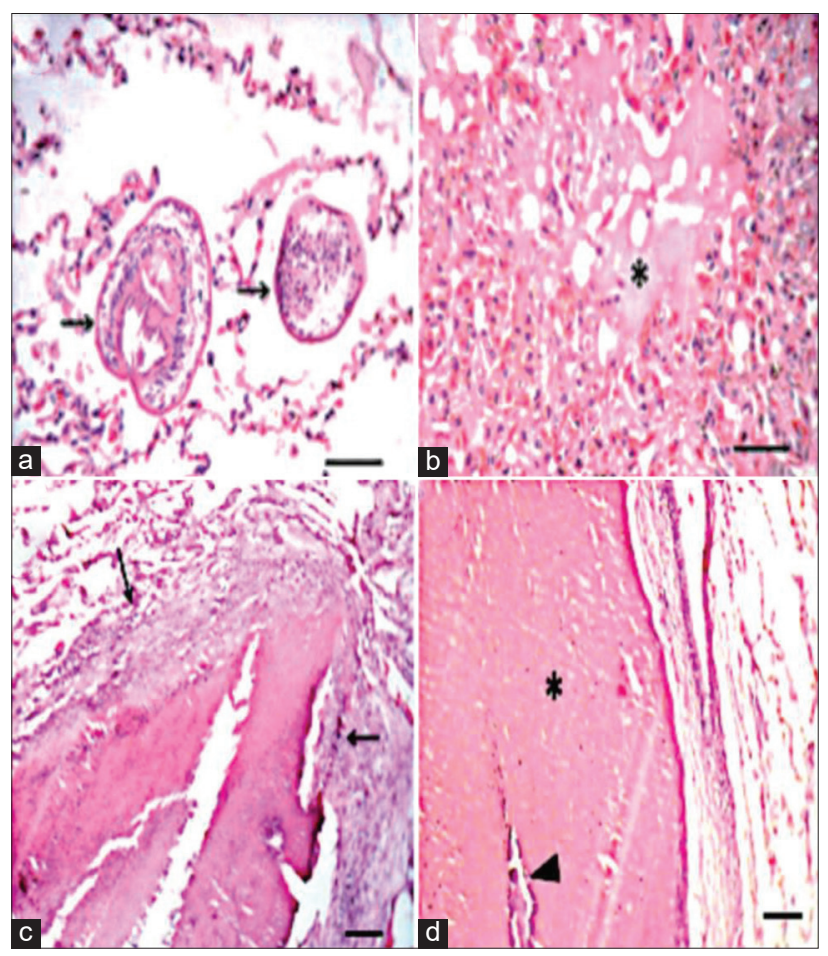

Figure-2: Lung of hydatid cyst infected camel showing: (a) Presence of scoleces (arrows) in alveoli, Bar $=50 \mu \mathrm{m}$, (b) pulmonary edema (asterisk), Bar $=50 \mu \mathrm{m}$, (c) suppurated cyst (abscess), Bar $=100 \mu \mathrm{m},(\mathrm{d})$ caseated (asterisk) and calcified (arrowhead) cyst, Bar $=100 \mu \mathrm{m}$.

serum level was significantly $(\mathrm{p}<0.05)$ lower than that of non-infected sheep. Hydatid infected cattle demonstrated a significantly $(\mathrm{p}<0.05)$ higher serum level of Th-1 pro-inflammatory cytokine, TNF- $\alpha$ and IL-6, comparing to non-infected cattle. However, Th-2 anti-inflammatory cytokines (IL-4 and IL-10) serum level was significantly lower $(p<0.05)$ than that of non-infected cattle. Data revealed that Th1polarized cytokines (TNF- $\alpha$ and IL-6) serum levels were significantly higher $(\mathrm{p}<0.05)$ in hydatid infected camel comparing to non-infected (Table-1).

\section{Electrophoretic and immunoreactive bands profile of hydatid cyst}

Overall polypeptide bands exhibited by the different prepared antigens; HCP-g, HCF-g, HCG-g, HCP-v, HCF-v, and HCG-v were 7, 3, 5, 16, 2, and 5 bands in number at MW 270, 103, 68.7, 47.7, 37.5, 32.8 , and $16 \mathrm{kDa} ; 65.8,46.0$, and $16.2 \mathrm{kDa} ; 98.9,70.7$, 54.0, 45.6, and $32.1 \mathrm{kDa} ; 270,250.9,223,175,151.3$, 112.1, 97.6, 70.9, 55.7, 48.1, 45.5, 41, 38.5, 36.4, 32, and $23 \mathrm{kDa} ; 70.9$, and $20 \mathrm{kDa}$; and 105.1, 73.2, 55.8, 46.5 , and $31.8 \mathrm{kDa}$, respectively (Figure-3). The resultant data revealed four shared protein bands at MW 270, 32, 45, and 46 between HCP-g and HCP-v; HCP-g, HCG-g, HCP-v, and HCG-v; HCG-g and HCP-v; and HCF-g and HCG-v, respectively (Figure-3).

The reactive band profiles presented by binding of specific $\operatorname{IgG}$ in positive naturally infected sera against hydatid cyst antigens; HCP-g, HCF-g, HCG-g, HCP-v, and HCG-v were identified at MW $270,190,94.7,70.3,62.5,56,50.6,48,45,42,38.1$, and $20.1 \mathrm{kDa} ; 103.2,71.5,59.1,54.7,41,29.1$, and $21.8 \mathrm{kDa} ; 99,82.6,50.8,36.7,32,31.2,19.5$, and $16 \mathrm{kDa} ; 270,155.2,129.6,122.6,102.5,83.7,79.7$, $65.8,55.8,45,42,40.7,37.5,32$, and $17.9 \mathrm{kDa}$; and $100.4,83.5,50.5,32$, and $16 \mathrm{kDa}$, respectively. However, HCF-v did not show any reaction with naturally infected sera (Figure-4). The shared immune reactive polypeptides were detected at MW 50, 45, 42, and $32 \mathrm{kDa}$ among HCP-g, HCG-g, and HCG-v; HCP-g and HCP-v; HCP-g and HCP-v; and HCG-v, HCP-v, and HCG-g, respectively (Figure-4).

The reactive profile of immunogenic bands presented when such hydatid cyst antigens of camel origin verified against pooled positive naturally infected cattle and sheep sera revealed the immunoreactive 
band at MW $32 \mathrm{kDa}$ with $\mathrm{HCP}-\mathrm{g}$ antigen, while HCP-v antigen showed the bands at MW 50, 31, and $30 \mathrm{kDa}$ and 53, 33, and $32 \mathrm{kDa}$ when using positive naturally infected cattle and sheep sera, respectively (Figure-5a and b).

\section{Diagnostic accuracy of HCP-g antigen}

HCP-g antigen was evaluated in the detection of hydatid cyst infection in collected camel sera using indirect ELISA. The used antigen achieved $75 \%$ sensitivity and a limited specificity $33 \%$. The apparent prevalence of hydatidosis in tested serum samples was $69 \%$.

\section{Detection of the specific IgG levels}

The total IgG level was determined to detect the humoral antibody responses to echinococcal infection. The IgG level was high in all infected animals in comparison with the non-infected ones (Figure-6).

\section{Discussion}

E. granulosus could be considered as a basic cause of high rates of liver and lung condemnation in slaughtered farm animals [23]. Urgent insights into host-metacestode interaction, immune evasion and immunopathogenesis of larval metacestode (hydatid cyst formation) are needed for diagnostic improvement and monitoring progression/regression of this disease.

In the current study, the lungs of camels had marked hydatid cysts which consisted of the outer laminated layer and fibrous layer penetrated with inflammatory cells. The surrounding pulmonary alveoli were collapsed, and some were emphysematous. Scoleces were found in alveoli with mononuclear inflammatory cells and few multinucleated giant cells aggregation. The cellular reaction associated with hydatid cysts was indicative of delayed hypersensitivity reactions [24]. The observed lesions associated with hydatid cysts in the examined lung tissues were similar to those reported before [23].

The echinococcal infection can stimulate or suppress immune responses, which persist and flourish for a long time in their mammalian hosts [25]. Th1 and Th2 cytokines induced different immune pathways to fight parasitic infections; Th1 cytokines coordinate cellular immune responses, and Th2 cytokines coordinate humoral immune responses [12]. In the

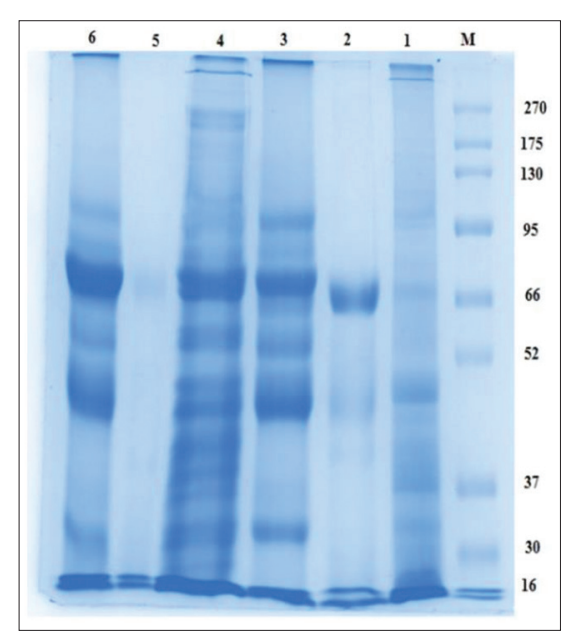

Figure-3: Sodium dodecyl sulfate-polyacrylamide gel electrophoresis electrophoretic protein profile of hydatid cyst protoscoleces-lung camel origin (HCP-g, Lane 1), hydatid cyst fluid-lung camel origin (HCF-g, Lane 2), hydatid cyst germinal layer-lung camel origin (HCG-g, Lane 3), hydatid cyst protoscoleces -liver camel origin ( $\mathrm{HCP}-\mathrm{v}$, Lane 4), hydatid cyst fluid-liver camel origin (HCF-V, Lane 5), hydatid cyst germinal layer-liver camel origin (HCG-V, Lane 6), and BLUltra prestained protein ladder, GeneDirex (Lane M, 16-270 kDa).

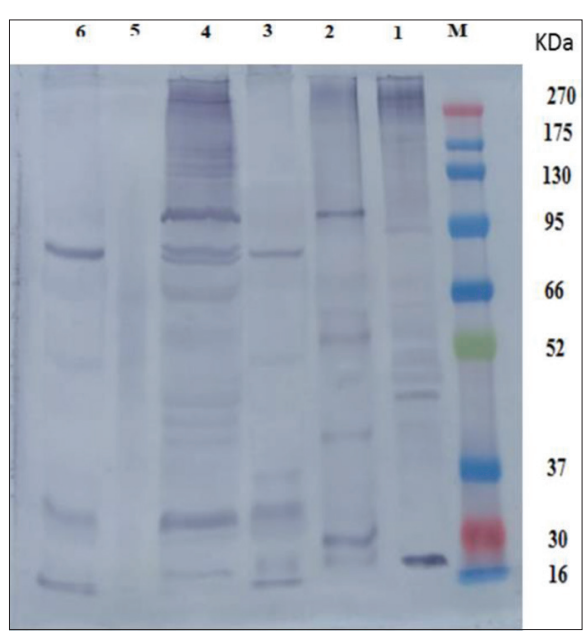

Figure-4: Western blot analysis profile of hydatid cyst protoscoleces-lung camel origin ( $\mathrm{HCP}-\mathrm{g}$, Lane 1), hydatid cyst fluid-lung camel origin (HCF-g, Lane 2), hydatid cyst germinal layer-lung camel origin (HCG-g, Lane 3), hydatid cyst protoscoleces-liver camel origin ( $\mathrm{HCP}-\mathrm{v}$, Lane 4), hydatid cyst fluid-liver camel origin (HCF-v, Lane 5 ), hydatid cyst germinal layer-liver camel origin (HCG-v, Lane 6) against pooled positive naturally infected camel sera and BLUltra prestained protein ladder, GeneDirex (Lane M, 16-270 kDa).

Table-1: Serum levels of Th1-polarized cytokines (TNF- $\alpha$ and IL-6) and Th2-polarized cytokines (IL-4 and IL-10) in hydatid infected-sheep cattle and camels $(\mathrm{ng} / \mathrm{ml})$.

\begin{tabular}{|c|c|c|c|c|c|c|}
\hline \multirow[t]{2}{*}{ Parameter/Animals } & \multicolumn{2}{|c|}{ Sheep } & \multicolumn{2}{|c|}{ Cattle } & \multicolumn{2}{|c|}{ Camel } \\
\hline & Non-infected & $\begin{array}{c}\text { Hydatid cyst } \\
\text { infected }\end{array}$ & Non-infected & $\begin{array}{l}\text { Hydatid cyst } \\
\text { infected }\end{array}$ & Non-infected & $\begin{array}{l}\text { Hydatid cyst } \\
\text { infected }\end{array}$ \\
\hline $\mathrm{NF}-\alpha$ & $8.41 \pm 1.24$ & $12.04 \pm 0.712^{*}$ & $9.29 \pm 0.647$ & $13.844 \pm 0.316^{*}$ & $10.76 \pm 0.289$ & $16.84 \pm 0.316 * *$ \\
\hline IL-6 & $9.10 \pm 0.51$ & $13.62 \pm 0.954 *$ & $7.83 \pm 0.587$ & $10.361 \pm 1.370 *$ & $8.38 \pm 0.741$ & $13.39 \pm 0.459 * *$ \\
\hline $\mathrm{IL}-4$ & $9.76 \pm 0.873$ & $6.39 \pm 0.647 *$ & $13.29 \pm 0.734$ & 8. $472 \pm 0.312 *$ & $12.78 \pm 0.548$ & $10.84 \pm 0.975$ \\
\hline IL-10 & $11.32 \pm 0.755$ & $9.57 \pm 0.289$ & $16.28 \pm 1.281$ & $10.589 \pm 0.921 *$ & $11.48 \pm 0.673$ & $9.81 \pm 0.762$ \\
\hline
\end{tabular}

All data expressed as Mean \pm SE. * and **Significantly different than non-infected group at $\mathrm{p}<0.05$ and $\mathrm{p}<0.01$, respectively. TNF- $\alpha=$ Tumor necrosis factor-alpha, IL=Interleukin 


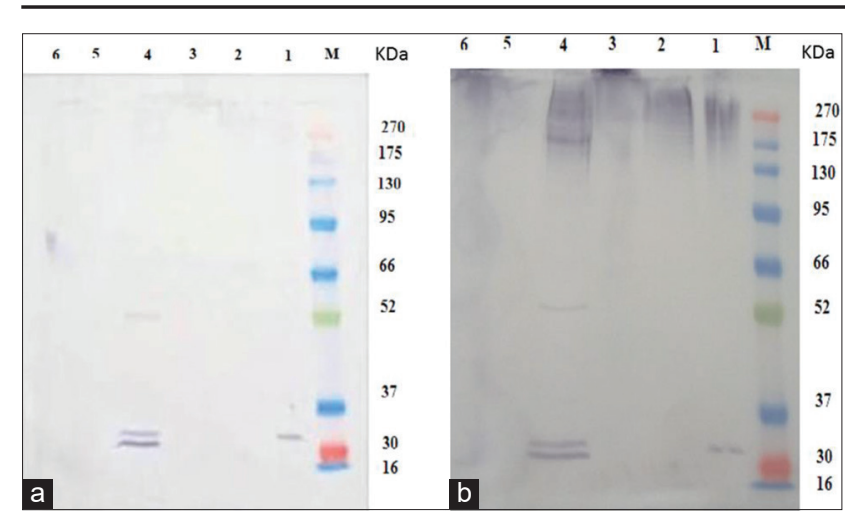

Figure-5: Western blot analysis profile of immunogenic bands of hydatid cyst antigens from camel origin against positive naturally infected cattle (a) and sheep sera (b). Hydatid cyst protoscoleces-lung camel origin (HCP-g, Lane $1 a$ and $b$ ), hydatid cyst fluid-lung camel origin (HCF-g, Lane $2 a$ and $b$ ), hydatid cyst germinal layer-lung camel origin ( $\mathrm{HCG}-\mathrm{g}$, Lane $3 a$ and $b$ ), hydatid cyst protoscolecesliver camel origin ( $\mathrm{HCP}-\mathrm{v}$, Lane $4 \mathrm{a}$ and $\mathrm{b})$, hydatid cyst fluid-liver camel origin (HCF-v, Lane $5 a$ and b), hydatid cyst germinal layer-liver camel origin (HCG-v, Lane 6a and b) against pooled positive naturally infected cattle (a) and sheep (b) sera and BLUltra prestained protein ladder, GeneDirex (Lane M, 16-270 kDa).

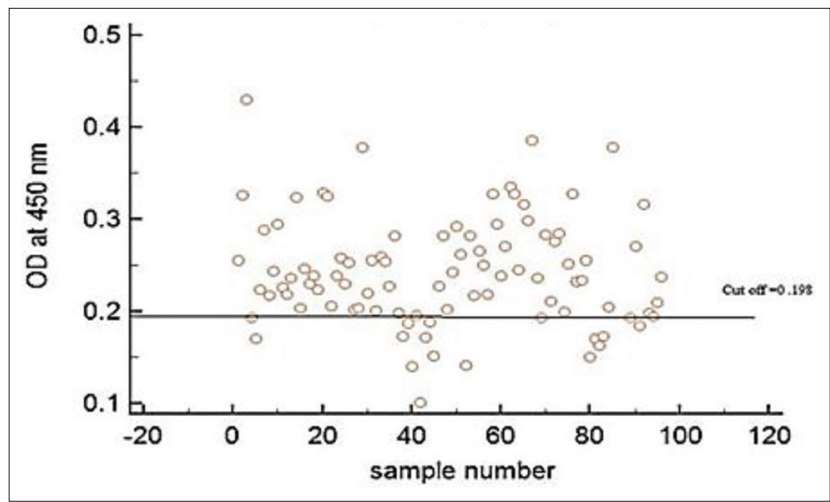

Figure-6: Total immunoglobulin G (IgG) antibody responses against a crude antigen preparation from Echinococcus granulosus protoscoleces was detected by an indirect enzyme-linked immunosorbent assay. The cutoff is the mean $\pm 3 \mathrm{SD}$ of negative sera from non-infected camels. The figure illustrates elevation in the level of IgG within naturally infected animals in comparison with non-infected ones.

current study, the serum levels of Th-1 pro-inflammatory cytokines, TNF- $\alpha$ and IL-6, in infected sheep, cattle, and camel were significantly higher in comparison with their levels in non-infected ones. However, Th-2 anti-inflammatory cytokines, IL-4 and IL-10, serum levels in infected sheep, cattle, and camel were lower than that of non-infected ones. These results showed a pattern of increase in Th1-type cytokines and decrease in a Th2-type cytokine. This agreed with results of Rostami-Rad et al. [14] which revealed a pattern of increase in Th1-type cytokines and decrease in a Th2-type cytokine after protoscoleces inoculation in experimentally infected mice at the beginning of the infection, however, after several weeks post-infection, Th1-type cytokines decreased and a Th2-type cytokine increased. Furthermore, these results are consistent with former studies that implied the concurrent activation of type 1 and type 2 cytokines in response to hydatid antigens [10]; however, the predominance of Th2 profiles in peripheral immune reactions may be reported in found hydatid infection in human hosts [26]. This could be attributed to dominant Th1 cytokines usually found to be associated with the killing and clearance of protoscoleces [7]. As well, regulatory mechanisms which are elevated gradually with the prolonged chronicity are responsible for inhibition of metacestode killing and immunoregulation of effector cells to limit the inflammatory responses [13] as it is able to control both Th1 and Th2 parasite killing effector mechanisms [11].

Modulation of the host immune response may also be relevant for selection toward commensalism, since it may prevent deleterious effects to the host resulting from the exacerbated immune response [27]. In general, the presented results showed a mixed coexistence of Th1 and Th2 with largely Th1 dominance. The immunological events related to this type are characterized by Th-1 polarized cytokine which is involved in initiating calcification [28] with a high level of macrophages at host pericyst layer [29]. The presented result confirmed this observation as showed a calcified cyst with macrophages infiltration in the surrounding tissue of the hydatid cyst in infected animals. However, the presence of eosinophils was able to kill protoscoleces with Th2 polarized cytokine [30]. The coexistence of Th1 and Th2 response is a remarkable feature of echinococcal infection. This is maybe due to the presence of different $\mathrm{CE}$ antigens with distinct epitopes to both types of T cells. Cell-mediated immunity is a characteristic feature of echinococcal infection [26,31].

In the present study, a comprehensive investigation to immunoreactive protein expression patterns of different prepared hydatid cyst antigens of camel origin; HCP-g, HCF-g, HCG-g, HCP-v, HCF-v, and HCG-v are electro-blotted against their homologous serum (positive naturally infected-hydatid cyst camel) and heterologous sera of positive naturally infected-hydatid cyst cattle and sheep sera. The resultant data revealed that HCP-g antigen could be considered a representative antigen for all other prepared antigens, where, such antigen shared them in one or more immune reactive polypeptide bands detected at MW 50, 45, 42, and $32 \mathrm{kDa}$ among HCP-g, HCG-g, and HCG-v; HCP-g and HCP-v; HCP-g and HCP-v; and HCG-v, HCP-v, and HCG-g, respectively, using their homologous serum. Moreover, Burgu et al. [32] blotted HCF from sheep origin and tested it against sheep infected sera found immune responses to antigenic bands at $116 \mathrm{kDa}$. This difference may be attributed to the difference in hydatid cyst origin, different quantity and quality of used chemical reagents [33].

The results revealed a limited specificity (33\%), which could be attributed to the failure of HCP-g antigen in the detection of true negative animal cases in non-infected hydatid cyst camels' sera. In addition, 
HCP-g antigen recorded a good sensitivity $(75 \%)$ and apparent prevalence $(69 \%)$ which may be attributed to the common shared immunoreactive bands which exhibited at MW $32 \mathrm{kDa}$ by HCP-g antigen and may be responsible for detecting high number of true positive cases in parasitological infected hydatid cyst camels' sera. Similar results were obtained by El-Shanawany et al. [34] who found that the immunoreactive bands of HCG from camel origin were 30 , 49,77 , and $99 \mathrm{kDa}$ and this was similar to the current study in finding $32 \mathrm{kDa}$ reacted with naturally camel serum sample. As well as, the immunodominant protein band at MW $32 \mathrm{kDa}$ was detected with HCP-g antigen using the heterologous sera against all different prepared hydatid cyst antigens of camel origin. This representative trait of HCP-g antigen could be attributed to the life stage universality dynamic nature of protoscoleces proteomic analysis [35] where our present results revealed the presence of the same shared immunoreactive band at $32 \mathrm{kDa}$ with $\mathrm{HCP}-\mathrm{v}$ antigen when the heterologous serum of sheep origin was used. Consequently, these data explore the potentiality of HCP-g antigen to be verified as a candidate vaccinal antigen to overcome hydatidosis in farm animals with great concern to the band at MW $32 \mathrm{kDa}$ which could also be used as a protein probe for sensitive detection of CE in herbivorous animals.

The current results indicated an elevation in the total IgG levels in infected camels comparing with non-infected ones. Furthermore, Rostami-Rad et al. [14], the total IgG level started to increase after 2 weeks' post-inoculation in experimentally infected mice, which is a representative of the humoral response against the parasite. In general, echinococcal infection could lead to activation of the humoral immune response in the form of a high level of $\mathrm{IgG}$, $\operatorname{IgM}$, and $\operatorname{IgE}[10]$.

\section{Conclusion}

CE naturally infected farm animals developed cellular and humoral immune responses. The results showed a pattern of increase in Th- 1 type cytokines and decrease in Th-2 type cytokines. This could explain that $\mathrm{CE}$ is a constant disease and confirm the reactivity Th1 in combating E. granulosus infection. In addition, echinococcal infection could lead to activation of the humoral immune response in the form of a high level of IgG.

\section{Authors' Contributions}

FAMA designed the study, performed the immunological analysis, and contributed to laboratory work analysis, statistics, data interpretation, manuscript preparation, and corresponded the authorship. SHMH contributed to antigens preparation, gel electrophoresis and Western blotting analysis with their interpretation. SSO performed the histopathological examination. DA shared in the preparation of antigens. EEE shared in gel electrophoresis and Western blotting analysis.
All authors assisted in manuscript preparation and have read and approved the final manuscript.

\section{Acknowledgments}

This work is a part of a project financially supported by the National Research Centre, Cairo, Egypt (project number 11020203).

\section{Competing Interests}

The authors declare that they have no competing interests.

\section{Publisher's Note}

Veterinary World remains neutral with regard to jurisdictional claims in published institutional affiliation.

\section{References}

1. Neumayr, A., Tamarozzi, F., Goblirsch, S., Blum, J. and Brunetti, E. (2013) Spinal cystic echinococcosis a systematic analysis and review of the literature: Part 1. Epidemiology and anatomy. PLOS Negl. Trop. Dis., 7(9): e2450.

2. Aboelhadid, S.M., El-Dakhly, K.M., Yanai, T., Fukushi, H. and Hassanin, K.M. (2013) Molecular characterization of Echinococcus granulosus in Egyptian donkeys. Vet. Parasitol., 193(1): 292-296.

3. Higuita, N.I.A., Brunetti, E. and McCloskey, C. (2016) Cystic echinococcosis. J. Clin. Microbiol., 54(3): 518-523.

4. Thompson, R. (2017) Biology and systematics of echinococcus. Adv Parasitol., 95:65-109.

5. Despommier, D.D. and Karapelou, J.W. (2012) Parasite Life Cycles. Springer Science and Business Media, Netherlands.

6. Jawad, A.R., Sulbi, I., Jameel, Y. and Ali, H. (2018) Molecular detection of Echinococcus granulosis from visceral organs of cattle abattoirs Kerbala province. J. Pure Appl. Microbiol., 12(2): 701-704.

7. Siracusano, A., Delunardo, F., Teggi, A. and Ortona, E. (2012) Host-parasite relationship in cystic echinococcosis: An evolving story. Clin. Dev. Immunol., 2012: Article ID 639362.

8. Rogan, M.T., Bodell, A.J. and Craig, P.S. (2015) Postencystment/established immunity in cystic echinococcosis: Is it really that simple? Parasite Immunol., 37(1): 1-9.

9. da Silva, M.A. (2011) Hydatid cyst/cystic echinococcosis: Anatomical and surgical nomenclature and method to quantify the cyst content solidification. Chin. Med. J., 124(18): 2806-2812.

10. Zhang, W., Wen, H., Li, J., Lin, R. and McManus, D.P. (2012) Immunology and immunodiagnosis of cystic echinococcosis: An update. Clin. Dev. Immunol., 2012: Article ID 101895 .

11. Tamarozzi, F., Mariconti, M., Neumayr, A. and Brunetti, E. (2016) The intermediate host immune response in cystic echinococcosis. Parasite Immunol., 38(3): 170-181.

12. Abo-Aziza, F.A.M., Hendawy, S.H.M., Namaky, A.H.E. and Ashry, H.M. (2017) Th1/Th2 balance and humoral immune response to potential antigens as early diagnostic method of equine strongylus nematode infection. Vet. World, 10(6): 679-687.

13. Díaz, Á. (2017) Immunology of cystic echinococcosis (hydatid disease). Br. Med. Bull., 124(1): 121-133.

14. Rostami-Rad, S., Jafari, R. and Darani, H.Y. (2018) Th1/ Th2-type cytokine profile in C57 black mice inoculated with live Echinococcus granulosus protoscolices. J. Infect. Public. Health, 11(6): 834-839.

15. Barghash, S., El Sayed, R., El-Alfy, N., Abou-Elnour, B., El-Kattan, A. and Ahmes, A.S. (2018) Prevalence and molecular identification of Echinococcus granulosus in 
humans and slaughtered animals in Egypt. Eur. J. Biomed. Pharm. Sci., 4(9): 34-42.

16. Bancroft, J.D. and Gamble, M. (2002) Theory and Practice of Histological Techniques. $6^{\text {th }}$ ed., Vol. 5. Churchill Livingstone, Elsevier, China. p130-175.

17. Tabar, G.H. and Razmi, G.R. (2009) Antibody response against hydatid fluid, protoscolex and whole body of Echinococcus granulosus antigens in lambs. Iran. J. Vet. Res., 10(3): 283-288.

18. Lowry, O.H., Rosebrough, N.J., Farr, A.L. and Randall, R.J. (1951) Protein measurement with the folin phenol reagent. J. Biol. Chem., 193(1): 265-275.

19. Laemmli, U.K. (1970) Cleavage of structural proteins during the assembly of the head of bacteriophage T4. Nature, 227(5259): 680-685.

20. Towbin, H., Staeheline, T. and Gordon, J. (1979) Electrophoretic transfer of proteins from polyacrylamide gels to nitrocellulose sheets: Procedure and some applications. Proc. Natl. Acad. Sci., 76: 4350-4354.

21. Oldham, G. (1983) Antibodies to Fasciola hepatica antigens during experimental infections in cattle measured by ELISA. Vet. Parasitol., 13(2): 151-158.

22. Almazán, C., Avila, G., Quiroz, H., Ibarra, F. and Ochoa, P. (2001) Effect of parasite burden on the detection of Fasciola hepatica antigens in sera and feces of experimentally infected sheep. Vet. Parasitol., 97(2): 101-112.

23. Abo-Aziza, F.A.M., Oda, S.S., Aboelsoued, D., Farag, T.K. and Almuzaini, A.M. (2019) Variabilities of hydatidosis in domestic animals slaughtered at Cairo and Giza abattoirs, Egypt. Vet. World, 12(7): 998-1007.

24. Singh, B., Singh, N.P., Murphy, E.A., Price, R.L., Fayad, R., Nagarkatti, M. and Nagarkatti, P.S. (2016) Histopathological changes associated with E. granulosus echinococcosis in food producing animals in Punjab (India). J. Parasit. Dis., 40(3): 997-1000.

25. Ahlberg, V. (2012) Echinococcus multilocularis immunomodulatory strategies in the intermediate host. Vet. Program., 71:1-10

26. Zhang, W., Ross, A.G. and McManus, D.P. (2008) Mechanisms of immunity in hydatid disease: Implications for vaccine development. J. Immunol., 181(10): 6679-6685.

27. Rossi, A., Marqués, J.M., Gavidia, C.M., Gonzalez, A.E., Carmona, C., García, H.H. and Chabalgoity, J.A. (2012) Echinococcus granulosus: Different cytokine profiles are induced by single versus multiple experimental infections in dogs. Exp. Parasitol., 130(2): 110-115.

28. Wu, X.W., Chen, X.L., Zhang, S.J., Zhang, X., Hong, S. and Peng, X.Y. (2011) Pericyst may be a new pharmacological and therapeutic target for hydatid disease. Chin. Med.J. (Engl)., 124(18): 2857-2862.

29. Peng, X., Li, J., Wu, X., Zhang, S., Niu, J., Chen, X., Yao, J. and Sun, H. (2006) Detection of osteopontin in the pericyst of human hepatic Echinococcus granulosus. Acta Trop., 100(3): 163-171.

30. Shamri, R., Xenakis, J.J. and Spencer, L.A. (2011) Eosinophils in innate immunity: An evolving story. Cell Tissue Res., 343(1): 57-83.

31. Hidalgo, C., Stoore, C., Strull, K., Franco, C., Corrêa, F., Jiménez, M., Hernández M., Lorenzatto, K., Ferreira, H.B., Galanti, N. and Paredes, R. (2019) New insights of the local immune response against both fertile and infertile hydatid cysts. PLoS One, 14(1): e0211542.

32. Burgu, A., Doganay, A., Gönenç, B., Sarimehmetoglu, H.O. and Kalinbacak, F. (2000) Analysis of fluids of hydatid cysts from sheep by SDS-PAGE, and determination of specific antigens in protein structure by western blotting. Turk. J. Vet. Anim. Sci., 24(5): 493-500.

33. Juyi, L., Yan, J., Xiufang, W., Zhaoqing, Z., Junliang, L., Mingxing, Z. and Wei, Z. (2013) Analysis of the chemical components of hydatid fluid from Echinococcus granulosus. Rev. Soc. Bras. Med. Trop., 46(5): 605-610.

34. El-Shanawany, E.E., Toaleb, N. and Rahman, E.H.A. (2019) Hydatid cyst germinal layer purified glycoproteins for diagnosis of camel cystic echinococcosis. J. Vet. Sci., 8(2): 101-105.

35. Cui, S.J., Xu, L.L., Zhang, T., Xu, M., Yao, J., Fang, C.Y., Feng, Z., Yang, P.Y., Hu, W. and Liu, F. (2013) Proteomic characterization of larval and adult developmental stages in Echinococcus granulosus reveals novel insight into host-parasite interactions. J. Proteomics, 84(12): 158-175. 